\title{
Multireference Stochastic Coupled Cluster
}

\author{
Maria-Andreea Filip, ${ }^{*}$ Charles J C Scott, and Alex J W Thom* \\ Department of Chemistry, University of Cambridge, Cambridge, UK \\ E-mail: maf63@cam.ac.uk: ajwt3@cam.ac.uk
}

\begin{abstract}
We describe a modification of the stochastic coupled cluster algorithm that allows the use of multiple reference determinants. By considering the secondary references as excitations of the primary reference and using them to change the acceptance criteria for selection and spawning, we obtain a simple form of stochastic multireference coupled cluster which preserves the appealing aspects of the single reference approach. The method is able to successfully describe strongly correlated molecular systems using few references and low cluster truncation levels, showing promise as a tool to tackle strong correlation in more general systems. Moreover, it allows simple and comprehensive control of the included references and excitors thereof, and this flexibility can be taken advantage of to gain insight into some of the inner workings of established electronic structure methods.
\end{abstract}

\section{Introduction}

The study of strong correlation in electron systems has been an important theme in electronic structure theory in recent years, as it is present in a series of interesting chemical systems, such as radicals, excited states, transition states and dissociating bonds. ${ }^{1}$ In the presence of strong correlation, typically high-accuracy methods like coupled cluster often fail to correctly describe the system. This failure has been attributed to the decrease in quality of the Hartree-Fock wavefunction as a first-order representation of the system, as the static correlation present often leads to near-degeneracies in the Hilbert space which cannot be captured by a single-determinant wavefunction.

Coupled cluster (CC) theory ${ }^{2 / 3}$ has become the most popular ab initio approach to electronic structure calculations, as it provides good results for medium-sized weakly correlated systems, while maintaining sizeconsistency and scaling polynomially with system size. However, for strongly correlated systems, it requires consideration of high level excitors in order to correctly estimate the correlation energy ${ }^{\sqrt{4}}$ Since its computational costs scale as $O\left(N^{2 i+2}\right)$, where $i$ is the truncation level and $N$ is the system size, this limits its use to very small systems.

One way to circumvent this issue and accurately treat some strongly correlated systems has been to use multiple reference determinants. Today, the field of multireference coupled cluster is very broad, with numerous methods developed over the last forty years, falling broadly into two categories: particleconserving methods, which either use multiple cluster operators in a Jeziorski-Monkhorst ansat $^{5 \sqrt[512]{12}}$ or a single cluster operator,,$\frac{134}{24}$ and Fock-space methods, ${ }^{25}[32]$ which generate wavefunctions with different numbers of electrons. While some of these have been successful in capturing the correlation energies of various test systems, ${ }^{25133 \sqrt{37}}$ they are plagued by various size-consistency and intruder-state issues. 1729138,46

In recent years, conventional quantum chemical techniques have been successfully combined with stochastic wavefunction propaga- 
tion methods to improve computational performance. A prime example of this is the Full Configuration Interaction Quantum Monte Carlo (FCIQMC) method. ${ }^{47}$ While, like Full Configuration Interaction (FCI), FCIQMC scales exponentially with system size, it does so with a significantly lower prefactor. This has allowed the method, together with its initiator adaptation, $\underline{48}$ to successfully treat a variety of systems. $\frac{49}{51}$

A stochastic solution to the coupled cluster equations has also been implemented using Projector Monte Carlo. $\frac{52}{2}$ This Coupled Cluster Monte Carlo (CCMC) method reproduces deterministic $\mathrm{CC}$ results to within stochastic error bars, but only needs to store a small fraction of the Hilbert space, leading to significantly lowered memory and computational costs. Both FCIQMC and CCMC have recently been used in conjunction with conventional coupled cluster as a means to include selected higher-order clusters in a $\mathrm{CC}$ calculation, either iteratively or not. 53154

In this paper we describe an implementation of multireference coupled cluster (using a single-reference formalism similar to that of $\frac{14115}{}$ ) within the stochastic paradigm, which allows for very quick implementation of such methods. In the following section we give an overview of the CCMC method and in the third section we describe our implementation of multireference Coupled Cluster Monte Carlo (mrCCMC). The fourth and fifth sections then presents a series of results obtained using this method on known strongly correlated molecular systems. These results are discussed in the general context of multireference methods in section 5 and some conclusions are given in section 6.

\section{Stochastic Coupled Clus- ter}

In deterministic $\mathrm{CC}$, the wavefunction is represented by the exponential ansatz

$$
\Psi_{\mathrm{CC}}=e^{\hat{T}}\left|D_{0}\right\rangle
$$

where $\left|D_{0}\right\rangle$ is a reference wavefunction (usually the Hartree-Fock wavefunction),

$$
\hat{T}=\sum_{\mathbf{i}} t_{\mathbf{i}} \hat{a}_{\mathbf{i}}
$$

and $\hat{a}_{\mathbf{i}}$ are excitors - combinations of creation and annihilation operators (for example, a second order excited determinant $\left|D_{i j}^{a b}\right\rangle=$ $\hat{a}_{b}^{\dagger} \hat{a}_{a}^{\dagger} \hat{a}_{i} \hat{a}_{j}\left|D_{0}\right\rangle$, so $\left.\hat{a}_{i j}^{a b}=\hat{a}_{b}^{\dagger} \hat{a}_{a}^{\dagger} \hat{a}_{i} \hat{a}_{j}\right)$. If we group the excitors based on their excitation level relative to the reference, we can also write

$$
\hat{T}=\sum_{i} \hat{T}_{i}
$$

where $i$ is the excitation level. This wavefunction is equivalent to the FCI wavefunction if all possible excitors are included. In truncated CC, $\hat{T}$ is limited to only excitors of up to a certain excitation level. In order to obtain $t_{\mathbf{i}}$, the Schrödinger equation is projected onto each of the determinants $\left|D_{\mathbf{i}}\right\rangle$ (including the reference), leading to a series of coupled cluster equations to be solved:

$$
\left\langle D_{\mathbf{i}}|\hat{H}-E| \Psi_{\mathrm{CC}}\right\rangle=0,
$$

where $E$ is the energy of $\Psi_{\mathrm{CC}}$. The number and complexity of these equations increases with the highest excitation level considered.

These equations are equivalent to

$$
\left\langle D_{\mathbf{i}}|1-\delta \tau(\hat{H}-E)| \Psi_{\mathrm{CC}}\right\rangle=\left\langle D_{\mathbf{i}} \mid \Psi_{\mathrm{CC}}\right\rangle
$$

Since

$$
\left\langle D_{\mathbf{i}} \mid \Psi_{\mathrm{CC}}\right\rangle= \pm\left\langle D_{0}\left|\hat{a}_{\mathbf{i}}^{\dagger}\right| \Psi_{\mathrm{CC}}\right\rangle=t_{\mathbf{i}}+O\left(\hat{T}^{2}\right)
$$

this can be approximately recast in an iterative form as

$$
t_{\mathbf{i}}(\tau+\delta \tau)=t_{\mathbf{i}}(\tau)-\delta \tau\left\langle D_{\mathbf{i}}|\hat{H}-E| \Psi_{\mathrm{CC}}\right\rangle
$$

It is possible to obtain the solutions to these equations from the population dynamics of a set of 'excips' in Hilbert space. This is done by stochastically sampling the action of the Hamiltonian, described by two processes: spawning of an excip from $\left|D_{\mathbf{i}}\right\rangle$ onto another $\left|D_{\mathbf{j}}\right\rangle$ coupled to it by the action of the Hamiltonian (with prob- 
ability proportional to $\left.\left\langle D_{\mathbf{j}}|\hat{H}| D_{\mathbf{i}}\right\rangle\right)$ and death of excips on $\left|D_{\mathbf{i}}\right\rangle$ (with probability proportional to $\left.\left\langle D_{\mathbf{i}}|\hat{H}-S| D_{\mathbf{i}}\right\rangle\right)$. The 'shift' $S$ replaces the parameter $E$ in the stochastic coupled cluster equations. Finally, pairs of excips of opposite signs on the same excitor annihilate each other, which helps ensure that the algorithm converges

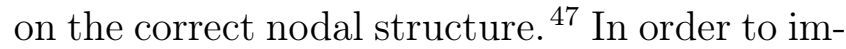
prove computational performance and stability, a series of modifications to this algorithm have been made, such as the deterministic selection of the reference and non-composite excitors, 56 the implementation of an efficient importancebased selection method, $\underline{57}$ the use of a similarity transformed Hamiltonian in the linked CCMC formalism $^{58}$ and the development of efficient excitation generators ${ }^{59 \mid 60}$ and parallelizable algorithms. ${ }^{56}$ More recently a diagrammatic version of CCMC has been implemented. $\underline{61}$

From a CCMC calculation, we have two estimators for the correlation energy of $\left|\Psi_{\mathrm{CCMC}}\right\rangle$ :

1. The instantaneous projected energy

$$
E_{\text {proj }}=\frac{\left\langle D_{0}|\hat{H}| \Psi_{\mathrm{CCMC}}\right\rangle}{\left\langle D_{0} \mid \Psi_{\mathrm{CCMC}}\right\rangle}
$$

2. The 'shift' $S$, which is expected to converge to the correlation energy once the calculation has reached a stable excip population. Once a target population has been reached in a CCMC population, the shift is set to vary starting from the instantaneous value of the projected energy. The shift is then updated every $A$ steps using 47

$$
S(\tau)=S(\tau-A \delta \tau)-\frac{\zeta}{A \delta \tau} \ln \frac{N_{\text {tot }}(\tau)}{N_{\text {tot }}(\tau-A \delta \tau)}
$$

where $N_{\text {tot }}$ is the total excip population.

\section{Multireference Coupled Cluster}

Multireference methods are justified by the desire to include "important" highly-excited determinants in the wavefunction expansion (e.g. configurations with many electrons in antibonding orbitals that acquire large coefficients during bond breaking). These are only included in the single reference $\mathrm{CC}$ (sr-CC) algorithm at high truncation levels. Their inclusion causes a significant improvement in the energy estimate (see Figure 4), but also requires an increased computational cost. However, by considering such determinants as part of the reference (or model) space of the calculation, they can be included without increasing the truncation level.

\subsection{Conventional MRCC}

Most Hilbert-space multireference coupled cluster methods are based on the JeziorskiMonkhorst formalism ${ }^{5}$

$$
\Psi_{\nu}=\sum_{\mu} c_{\nu \mu} e^{\hat{T}^{\mu}} \Phi_{\mu}
$$

where $\Phi_{\mu}$ are the reference-space functions, $\hat{T}^{\mu}$ are cluster operators defined relative to each of these references and $c_{\nu \mu}$ are CI coefficients. The formalism is the basis for so-called stateuniversal methods, where multiple wavefunctions are determined simultaneously. Statespecific methods have also been developed; here we will discuss a particular approach based on a single-reference formalism, known as SS $\operatorname{CCSD}(\mathrm{TQ})^{1415 / 6263}$ or CCSDtq. $\frac{16}{2}$ The starting point is to observe that for a given reference $\left|D_{\mathbf{i}}\right\rangle=\hat{a}_{i}\left|D_{0}\right\rangle$,

$$
e^{\hat{T}^{(\mathbf{i})}}\left|D_{\mathbf{i}}\right\rangle=e^{\hat{T}^{(\mathbf{i})}} \hat{a}_{\mathbf{i}}\left|D_{0}\right\rangle .
$$

It is therefore possible to rewrite any multireference wavefunction in terms of excitations of a single reference only. With the appropriate intermediate normalisation $\left(\left\langle D_{0} \mid \Psi\right\rangle=1\right.$, it is further possible to write it in an exponential form, $e^{\hat{T}^{\prime}} D_{0}$, where as before $\hat{T}^{\prime}=\sum_{i} \hat{T}_{i}^{\prime}$, but highly excited $\hat{T}_{i}^{\prime}$ no longer include all possible excitations of order $i$ from $\left|D_{0}\right\rangle$, but only those that can be reached by lower excitations from other references. To clarify, let us consider a complete $(2,2)$ reference space, given by the four determinants. $\left\{\left|D_{0}\right\rangle,\left|D_{I}^{A}\right\rangle,\left|D_{J}^{B}\right\rangle,\left|D_{I J}^{A B}\right\rangle\right\}$. $I$ and $A$ may be taken to be $\alpha$ spin-orbitals, and $J$ and $B$ as $\beta$. If we are interested in including up to double excitations out of this space in our cluster expansion, the wavefunction can be described as a single-reference exponential 
$\operatorname{ansatz}^{15}$ with

$$
\begin{aligned}
\hat{T}^{\prime} & =\hat{T}_{1}+\hat{T}_{2}+\sum_{I^{\prime}, j, k, a, b, C^{\prime}} \hat{T}_{3}\left(\begin{array}{c}
a b C^{\prime} \\
I^{\prime} j k
\end{array}\right) \\
& +\sum_{k, l, c, d, A, B} \hat{T}_{4}\left(\begin{array}{c}
c d A B \\
I J k l
\end{array}\right)
\end{aligned}
$$

where model space orbitals have been labelled with capital letters $I, J, K, \ldots A, B, C \ldots$ and general orbitals as $i, j, k \ldots a, b, c \ldots$. Primes have been used to differentiate summation indices over the model space from fixed model space orbitals.

In general, the cluster operator $\hat{T}^{\prime}$ can be written as a sum of internal and external cluster operators, 15

$$
\hat{T}^{\prime}=\hat{T}^{\mathrm{int}}+\hat{T}^{\mathrm{ext}}
$$

where $T^{\text {int }}$ gives excitations within the model space and $T^{\mathrm{ext}}$ produces excitations outside the model space.

$$
\begin{aligned}
& \hat{T}^{\mathrm{int}}=\sum_{I^{\prime}, A^{\prime}} \hat{T}_{1}\left(\begin{array}{c}
A^{\prime} \\
I^{\prime}
\end{array}\right)+\hat{T}_{2}\left(\begin{array}{c}
A B \\
I J
\end{array}\right) \\
& \hat{T}^{\mathrm{ext}}=\sum_{i, j, \ldots, a, b, \ldots} \hat{T}_{1}\left(\begin{array}{l}
a \\
i
\end{array}\right)+\hat{T}_{2}\left(\begin{array}{c}
a b \\
i j
\end{array}\right)+\ldots
\end{aligned}
$$

where at least one of the indices in each cluster in $\hat{T}^{\text {ext }}$ is not in the model space. The wavefunction may then be written as

$$
\Psi=e^{\hat{T}^{\mathrm{ext}}} e^{\hat{T}^{\mathrm{int}}}\left|D_{0}\right\rangle
$$

It is worth pointing out at this stage that the singly excited terms in the model space are constrained to have the correct spin (and therefore $\left|D_{I}^{B}\right\rangle$ and $\left|D_{J}^{A}\right\rangle$ are not included). However, pairs of model space orbitals of different spin can be included in $\hat{T}_{3}\left(\begin{array}{c}a b C^{\prime} \\ I^{\prime} j k\end{array}\right)$, so long as the overall spin of the terms is correct. In the case where all but two indices in the term come from outside the model space, such terms, while included in the ansatz, are not related by a less than double excitation to any of the model space determinants. This feature will be impor- tant when comparing to our stochastic method.

The ansatz can be generalised to include any excitation level $\lambda$ from a model space of all at most $k$-tuple excitations of a reference determinant, using a cluster operator ${ }^{15}$

$$
\begin{aligned}
\hat{T}^{\prime} & =\hat{T}_{1}+\ldots+\hat{T}_{\lambda}+\hat{T}_{\lambda+1}\left(\begin{array}{c}
a_{1} \ldots a_{\lambda} A_{1} \\
I_{1} i_{1} \ldots i_{\lambda}
\end{array}\right)+ \\
& +\ldots+T_{\lambda+k}\left(\begin{array}{c}
a_{1} \ldots a_{\lambda} A_{1} \ldots A_{k} \\
I_{1} \ldots I_{k} i_{1} \ldots i_{\lambda}
\end{array}\right)
\end{aligned}
$$

where indices $a_{1}, \ldots, a_{\lambda}, i_{1}, \ldots, i_{\lambda}$ correspond to active or inactive orbitals, while $A_{1}, \ldots, A_{k}$, $I_{1}, \ldots I_{k}$ are active indices. 


\subsection{Stochastic MRCC}

Consider a stochastic coupled cluster calculation with truncation level $m$. Currently, the single reference algorithm selects clusters that correspond to an excitation of up to order $m+2$ of the reference and allows spawning onto those that correspond to excitations up to order $m$. We introduce a secondary reference in this model by allowing spawning and selection to occur in an expanded space, of size and shape determined by this secondary reference. However, the clusters will still be described exclusively by their effect on the primary reference, so we will not need to consider propagation differently for the two references. For example, in a system where the secondary reference considered is an excitation of order $n$ of the primary reference, we allow clusters to be selected if they correspond to excitations up to order $n+m+2$ of the primary reference. For high separations between references, this requires sampling a significantly larger space than the single reference equivalent, but due to recent improvements to the selection algorithm, $\frac{57}{7}$ this can be done relatively efficiently. Spawning is then only allowed onto excitors within $m$ excitations of either of the references.

Figure 1 shows an example of two references 6 excitations apart, treated at CCSD level. While this model nicely highlights the relation between the selection and spawning spaces, for easier comparison to the conventional method of Piecuch, Oliphant and Adamowicz, $\frac{1415}{15}$ we will consider two references two excitations apart, $\left|D_{0}\right\rangle$ and $\left|D_{1}\right\rangle=\left|D_{I J}^{A B}\right\rangle$ and treat both at the CCSD level. The resulting wavefunction is given by $\Psi=e^{\hat{T}^{\prime}}\left|D_{0}\right\rangle$, where

$$
\begin{aligned}
\hat{T}^{\prime} & =\hat{T}_{1}+\hat{T}_{2}+\sum_{A^{\prime}, I^{\prime}, a, b, j, k, k, l}\left[\hat{T}_{3}\left(\begin{array}{c}
a A B \\
I J k
\end{array}\right)\right. \\
& \left.+\hat{T}_{3}\left(\begin{array}{c}
a b A^{\prime} \\
I J k
\end{array}\right)+\hat{T}_{3}\left(\begin{array}{c}
a A B \\
I^{\prime} j k
\end{array}\right)+\hat{T}_{4}\left(\begin{array}{c}
a b A B \\
I J k l
\end{array}\right)\right]
\end{aligned}
$$

where $A^{\prime}$ runs over model orbitals empty in $\left|D_{0}\right\rangle, I^{\prime}$ runs over occupied ones, $j, k, l$ are core orbitals and $a, b$ are virtual orbitals. In terms of internal and external cluster operators,

$$
\hat{T}^{\mathrm{int}}=\hat{T}_{2}\left(\begin{array}{c}
A B \\
I J
\end{array}\right)
$$

and $\hat{T}^{\mathrm{ext}}=\hat{T}^{\prime}-\hat{T}^{\mathrm{int}}$. Obviously,

$$
\hat{T}_{2}\left(\begin{array}{c}
A B \\
I J
\end{array}\right) \propto \hat{a}_{1},
$$

where $\hat{a}_{1}\left|D_{0}\right\rangle=\left|D_{1}\right\rangle$. Also if we label $\hat{T}^{(1)}$ cluster operators relative to $\left|D_{1}\right\rangle$, then

$$
\begin{aligned}
& \hat{T}_{3}\left(\begin{array}{c}
a A B \\
I J k
\end{array}\right) \propto \hat{T}_{1}^{(1)}\left(\begin{array}{l}
a \\
k
\end{array}\right) \hat{a}_{1} \\
& \hat{T}_{3}\left(\begin{array}{c}
a b A \\
I J k
\end{array}\right) \propto \hat{T}_{2}^{(1)}\left(\begin{array}{c}
a b \\
B k
\end{array}\right) \hat{a}_{1}
\end{aligned}
$$

and so on. We can therefore write

$$
\hat{T}^{\prime}=\hat{T}_{1}+\hat{T}_{2}+\left(\hat{T}_{1}^{(1)}+\hat{T}_{2}^{(1)}\right) \hat{a}_{1}
$$

taking care to only include overlapping contributions in the cluster operator relative to a single reference. This leads to multiple equivalent representation of $\hat{T}^{\prime}$ in this form. In our stochastic approach, we only consider each excited determinant once, regardless of which references and clusters it can be reached from, so these considerations are trivially avoided. This makes CCMC an ideal framework for this kind of algorithm, as it allows simple implementations of potentially complicated reference spaces. Generalizing to an arbitrary number of references, with arbitrary corresponding truncation levels, we obtain

$$
\hat{T}^{\prime}=\sum_{i=1}^{m_{0}} \hat{T}_{i}+\sum_{n=1}^{N} \sum_{j=0}^{m_{n}} \hat{T}_{j}^{(n)} \hat{a}_{n}
$$

where $\hat{T}_{i}$ are $i$-th order excitors of the first reference, $\hat{T}_{j}^{(n)}$ are $j$-th order excitors of the $n$-th secondary reference, $\hat{a}_{n}$ is the excitor that generates the $n$-th secondary reference from the first, $m_{n}$ is the truncation level for reference $n$ and $N$ is the number of secondary references used. We note here two differences from Eq. 17. Firstly, 
our formalism allows the definition of an arbitrary reference space, rather than requiring the inclusion of all excitations up to a certain order. Secondly, the truncation level with respect to each reference can be selected independently, allowing for additional flexibility.

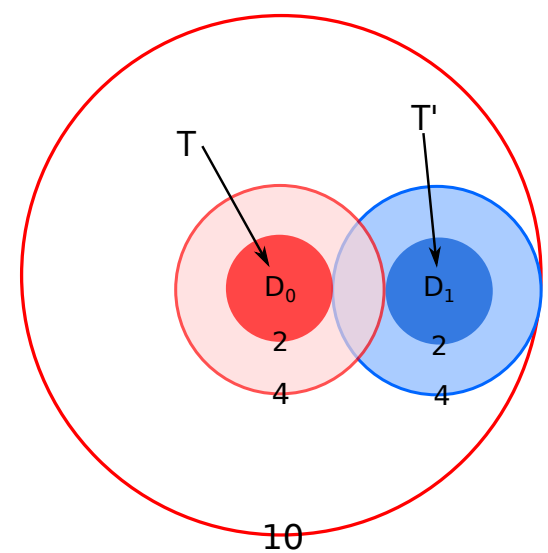

Figure 1: Example of the explored space of a multireference stochastic CCSD calculation with the two references 6 excitations apart. Clusters are selected within 10 excitations of $\left|D_{0}\right\rangle$ (inside the black circle), spawning attempts are made from clusters within 4 excitations of $\left|D_{0}\right\rangle$ or $\left|D_{1}\right\rangle$ (inside the transparent red/blue circles) to clusters within 2 excitations of $\left|D_{0}\right\rangle$ or $\left|D_{1}\right\rangle$ (inside the solid red/blue circles). The regular stochastic CCSD calculation would only explore the space inside the transparent red circle.

This algorithm effectively allows consideration of secondary references while maintaining the relative simplicity of the sr-CCMC approach. It is worth noting that, in a multireference calculation that explores the set of determinants within $m$ excitations of two references, there is an approximately twofold increase in the proportion of the Hilbert space that must be stored compared to the corresponding single-reference calculation, truncated at excitation level $m$. In general, we expect the space spanned by a calculation to increase at most linearly with the number of references, provided the truncation levels are the same for all references, as the spaces spanned by the cluster expansion about each reference may overlap, leading to slight sublinearity. In large basis sets, this is insignificant relative to the $\mathcal{O}\left(N^{2 n}\right)$ increase in memory costs associated with increasing the truncation level to $m+n$ in order to include the same determinants in a singlereference calculation. If lower truncation lev- els can be used to obtain results of the same accuracy, the scaling with system size is reduced polynomially. With the current selection scheme, the size of the selection space is only determined by the highest excited secondary reference, so we expect the computational scaling with number of references to be favourable.

If we include the two single excitations $\left|D_{I}^{A}\right\rangle$ and $\left|D_{J}^{B}\right\rangle$ in the model space, equation 118 becomes

$$
\begin{aligned}
\hat{T}^{\prime} & =\hat{T}_{1}+\hat{T}_{2}+\sum_{A^{\prime}, I^{\prime}, a, b, j, k, l}\left[\hat{T}_{3}\left(\begin{array}{c}
a A B \\
I J k
\end{array}\right)\right. \\
& +\hat{T}_{3}\left(\begin{array}{c}
a b B \\
J k l
\end{array}\right)+\hat{T}_{3}\left(\begin{array}{c}
a b A \\
I j k
\end{array}\right) \\
& \left.+\hat{T}_{3}\left(\begin{array}{c}
a b A^{\prime} \\
I J k
\end{array}\right)+\hat{T}_{3}\left(\begin{array}{c}
a A B \\
I^{\prime} j k
\end{array}\right)+\hat{T}_{4}\left(\begin{array}{c}
a b A B \\
I J k l
\end{array}\right)\right]
\end{aligned}
$$

If we compare this to equation (12), we find all terms are accounted for, except for those of the form $\hat{T}_{3}\left(\begin{array}{c}a b B \\ I k l\end{array}\right)$ and $\hat{T}_{3}\left(\begin{array}{c}a b A \\ J k l\end{array}\right)$. This is because, as mentioned as the end of the previous section, these are not within two excitations of any of the references. We therefore expect that, depending upon the magnitude of the contributions of such terms to the wavefunction, we may be able to observe differences between the SS CCSD(TQ) method and the mr-CCMCSD method, even when using the same model space. It is also worth pointing out that, while in our case there is a difference between equations (18) and (25), both of these model spaces would be described by the same cluster operator in SS CCSD(TQ), as the set of active orbitals is unchanged. Only the formal split of excitors between $\hat{T}^{\text {int }}$ and $\hat{T}^{\text {ext }}$ would change.

\section{Two-Reference Results}

\subsection{The $\mathrm{S}_{4}$ model}

First we look at a simple 4-electron system, known as the $\mathrm{S}_{4}$ model $-\mathrm{H}_{4}$ in a square geometry,. $\stackrel{41}{ }$ The symmetry of the system and the fact 
that each of the $\mathrm{H}-\mathrm{H}$ distances may be longer than an equilibrium $\mathrm{H}_{2}$ bond introduces significant electron correlation to the system, so we expect it to have some multireference character. As we increase the $\mathrm{H}-\mathrm{H}$ separation, while maintaining the square geometry, the amount of strong correlation in the system also increases.

In a minimal basis, $\frac{64}{6}$ this system only has 10 Slater determinants in its Hilbert space, so we can easily obtain the FCI energy. In this case, both CCSDTQ and mr-CCSD with two references (2r-CCMCSD), where the second reference is chosen to be the highest excited determinant, explore the entire Hilbert space, so we expect very good agreement of both methods with FCI. Therefore this system is a good test that the behaviour of our algorithm is as expected. We can see from Table 1 that, at $r_{\mathrm{HH}}=2 a_{0}$ there is indeed good agreement between the FCI result, CCMCSDTQ and 2rCCMCSD projected energies, with differences of less than 0.1 milliHartrees, well within chemical accuracy $\left(1.6 \times 10^{-3}\right.$ Hartree $)$. Our results compare favourably to conventional MRCC results obtained for this system. $\frac{34 \mid 41 / 42}{}$

Table 1: Values of the calculated correlation energy for $\mathrm{H}_{4}$ in a minimal basis.

\begin{tabular}{|c|c|}
\hline Method & Energy $/ \mathrm{E}_{h}$ \\
\hline FCI & -0.117621 \\
CCMCSD & $-0.12044(3)$ \\
CCMCSDT & $-0.12059(7)$ \\
CCMCSDTQ & $-0.11761(7)$ \\
2r-CCMCSD & $-0.11763(4)$ \\
MRCCSD $^{34}$ & -0.117580 \\
MRCCSD-1 $^{41}$ & -0.117686 \\
MRCCSD- $2,3^{41}$ & -0.117575 \\
MRACPQ- $^{42}$ & -0.117102 \\
\hline
\end{tabular}

A range of conventional MRCC methods ${ }^{41142}$ have been used to investigate this system in its strongly correlated regime $\left(\alpha / a_{0} \in[2,7]\right)$. As can be seen in Figure 2 the quality of our energy estimate remains consistent over this interval, showing an order of magnitude improvement over previous results.

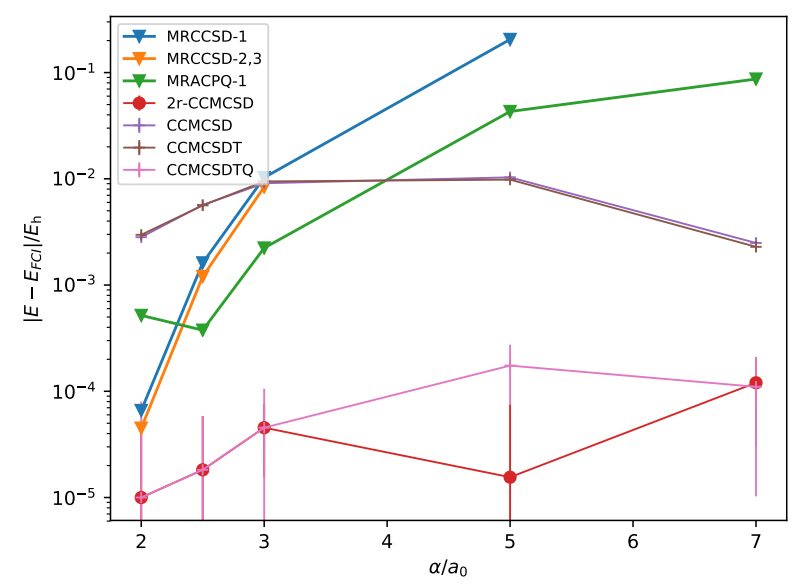

Figure 2: Difference in correlation energy captured by various single and multireference coupled cluster methods relative to FCI, as we increase the length of the side of the $\mathrm{H}_{4}$ square, $a$. As expected CCSDTQ and $2 \mathrm{r}-\mathrm{CCSD}$ are both in very good agreement with FCI over the full range of geometries investigated.

\subsection{The $\mathrm{N}_{2}$ molecule}

The next system of interest is $\mathrm{N}_{2}$, which is known to be difficult to accurately describe by single reference methods at stretched geometries, due to correlation effects caused by the dissociation of the triple bond. ${ }^{465}$ Going from the equilibrium bond length $\left(2.118 a_{0}\right)$ to $3.6 a_{0}$, the convergence of the coupled cluster energy with truncation level becomes significantly poorer (Figure 4), requiring costly, hightruncation level calculations to converge on the FCI result. mr-CCMC can be applied to this system, using a sixth order excitation of the Hartree-Fock determinant as our second reference. This corresponds to exciting six electrons from bonding $\sigma$ and $\pi$ orbitals to anti-bonding ones (see Figure 3). We expect that this determinant is crucial in describing the bond breaking that occurs as the nitrogen molecule is stretched and therefore a good candidate for a second reference.

The numerical results of single- and multireference calculations on stretched nitrogen are given in the Supporting Information. For reference, Hartree-Fock energies are also given.

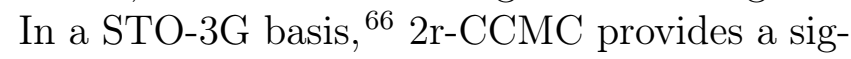
nificant improvement to our energy estimates, making 2r-CCMCSDT sufficient to get within chemical accuracy of the calculated FCI energy 


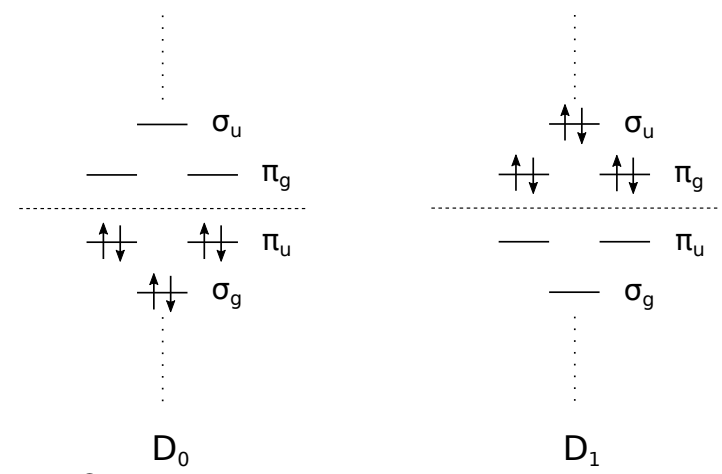

Figure 3: Active orbitals occupied in the two references used for the nitrogen molecules.
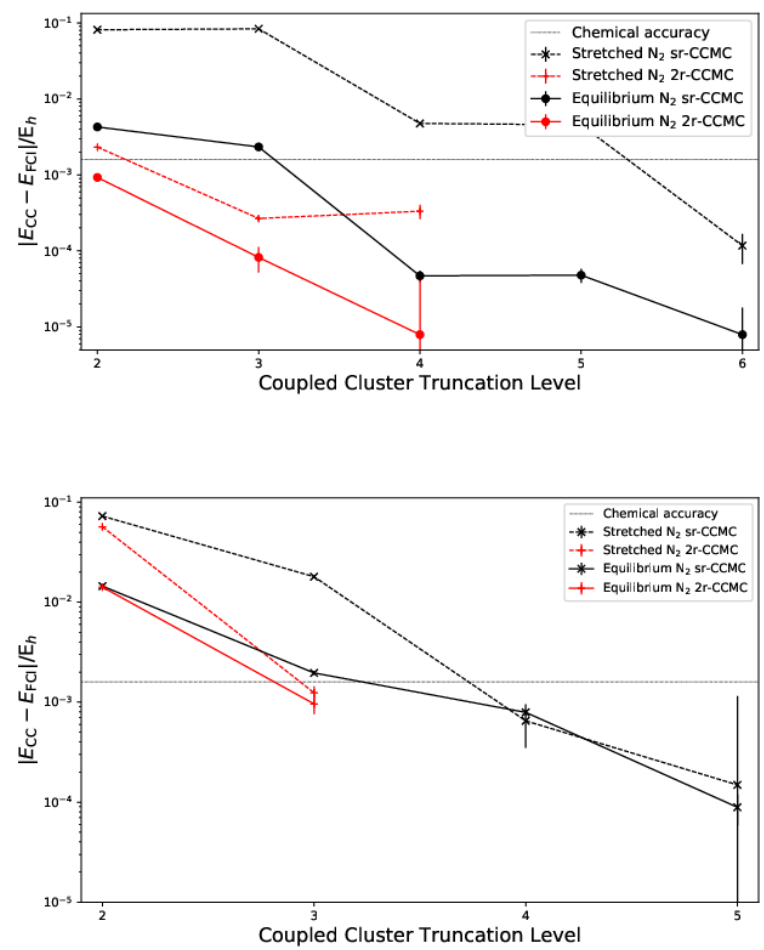

Figure 4: The difference between the coupled cluster and the FCI energy for different CC truncation levels for $\mathrm{N}_{2}$ in a minimal basis (top) and in a Dunning cc-pVDZ basis(bottom) with frozen core electrons. For the larger basis set, the shift was used as a correlation energy estimator rather than the projected energy, due to difficulties collecting statistics on the latter. While in the single-reference case high truncation levels are needed to obtain sub-milliHartree accuracy, 2r-CCMCSDT is sufficient to achieve this.

(Figure 4). A similar improvement can also be observed when treating the molecule in a larger Dunning cc-pVDZ basis set ${ }^{67}$ with frozen core electrons (Figure 4), confirming that the faster convergence is not simply a consequence of the multireference space effectively covering a high proportion of the relatively small STO3G Hilbert space.
Figure 5 shows the proportion of the Hilbert space populated after the system has reached steady-state for single and multireference calculations. In general, coupled cluster memory costs should scale as $\mathcal{O}\left(N^{2 l}\right)$ and even calculations with high truncation levels only use a small fraction of the full Hilbert space of the system. Stochastic methods decrease the memory cost by a constant pre-factor. ${ }^{55}$ It can be seen from Figure 5 that 2r-CCMC produces more accurate results at a reduced memory cost relative to single-reference CCMC. Also, if CCSDT can be used to obtain results of similar accuracy to CCSDTQ, this reduces the scaling with system size by a factor of $N^{2}\left(N^{6}\right.$ vs. $\left.N^{8}\right)$, provided an efficient sampling method for the multireference space is implemented.

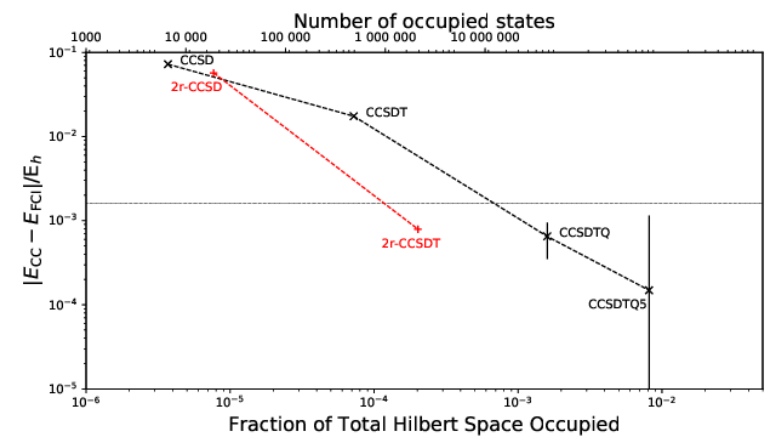

Figure 5: Convergence of CCMC energy versus the proportion of the total Hilbert Space that is populated once steady-state has been reached in Dunning cc-pVDZ stretched $\mathrm{N}_{2}$, with frozen core electrons. The points correspond to successive truncation levels, starting at CCSD. It can be seen that 2r-CCMC achieves higher accuracy results with only a fraction of the memory requirements of high-level single-reference calculations.

We have also used mr-CCMC to calculate a binding curve for $\mathrm{N}_{2}$, given in Figure 6. Curves obtained from 2r-CCMCSDT are in significantly better agreement with FCI values ${ }^{4}$ than CCSD or CCSDT. These results will be discussed further in the following section.

\subsection{The $\mathrm{N}_{3}^{-}$anion}

Finally, we look at the azide anion in order to assess the effect of using a second reference in systems with larger numbers of electrons. We have found that both the equilibrium geometry $\left(r_{\mathrm{NN}}=1.16 \AA\right)^{68}$ and a linear symmet- 


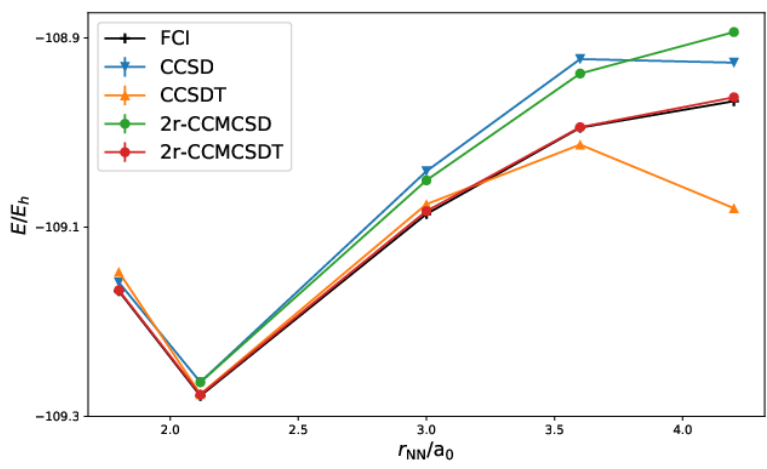

Figure 6: $\mathrm{N}_{2}$ binding curves obtained using the Dunning cc-pVDZ basis set. At large separations $\left(r=4.2 a_{0}\right)$, the two-reference solution is metastable, with a long enough lifetime to collect statistics. Single-reference CCMC calculations are shown aside from $r=4.2 a_{0}$ where deterministic CC values from Ref. 4 are given as the CCMC calculations are unstable, and $r=1.8 a_{0}$ where a deterministic CC calculation was performed. FCI results are from Ref. 4, except for at $r=1.8 a_{0}$, where an FCIQMC calculation was performed.

rically stretched geometry $\left(r_{\mathrm{NN}}=2.0 \AA\right)$ require high truncation levels for the CCMC energy to converge. For the multireference calculations, a quadruple excitation was used as the second reference, corresponding to the excitation of the four electrons in the non-bonding $\pi$ orbitals to the corresponding antibonding orbitals. As can be seen in Figure 7, once again 2r-CCMC provides a significant improvement to the energy estimate, even if 2r-CCMCSDT is not sufficient to reach chemical accuracy in the stretched case.

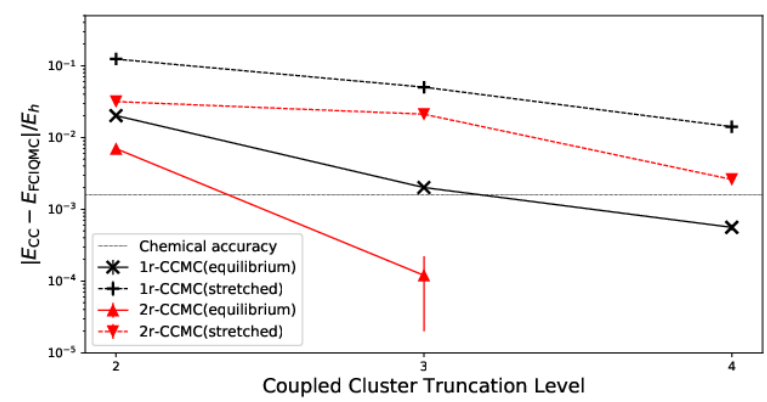

Figure 7: The difference between the CCMC and the FCIQMC energy for different CC truncation levels for $\mathrm{N}_{3}^{-}$in a minimal basis, at equilibrium and stretched geometries. Multireference CCMC provides a systematic improvement of the energy in both cases.

The poorer convergence for $2 \mathrm{r}-\mathrm{CCMC}$ for $\mathrm{N}_{3}^{-}$ suggests that the choice of secondary reference has a significant effect on the quality of the results. This is as expected, following from the notion that references should be highly weighted determinants in the expansion of the true ground state wavefunction. In the case of $\mathrm{N}_{2}$ we were aware of such a determinant, but for $\mathrm{N}_{3}^{-}$, we have at multiple reasonable choices of secondary reference, one of which is the fourth order excitation used. However, given that this excitation is already significant in the equilibrium geometry, it is likely that upon stretching the bonds, more highly excited determinants (perhaps the one corresponding to the excitation of both $\sigma$ and $\pi$ electrons, as for $\mathrm{N}_{2}$, or the excitation of bonding rather than nonbonding $\pi$ electrons) become highly weighted in the ground state and would therefore serve as better secondary references.

\section{Beyond two references}

We have shown in the previous section that, using two references, mr-CCMC is more successful in capturing the correlation in difficult molecular systems than the corresponding single reference methods. In this section we will turn our attention to the performance of the method relative to conventional MRCC methods and propose a procedure to balance the accuracy of our method against its computational cost.

\subsection{Comparison to conventional MRCC methods}

\subsubsection{The $\mathrm{H}_{8}$ model}

First, we turn our attention to the $\mathrm{H}_{8}$ model, shown in Figure 8, in a minimal basis. ${ }^{64}$ As the parameter $\alpha$ is varied from 0 to $\infty$, the degree of electron correlation in the system decreases, as it dissociates to 4 independent $\mathrm{H}_{2}$ molecules. The HOMO and LUMO of the system at $\alpha=a_{0}$ become closer in energy as we decrease $\alpha$, tending to degeneracy at $\alpha=0$. Therefore, these orbitals form a natural choice of model space for mr-CCMC. This system has been studied using the SS CCSD(TQ) method 


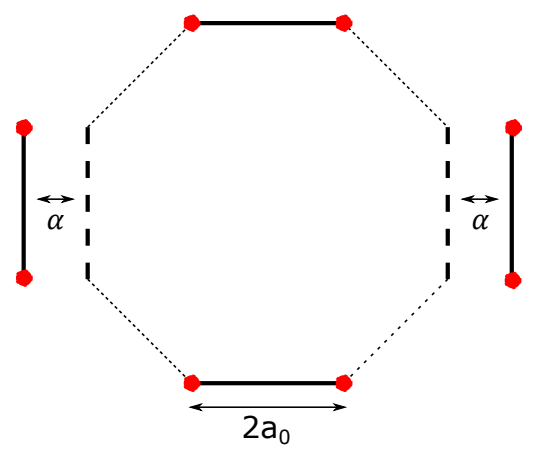

Figure 8: Geometry of the $\mathrm{H}_{8}$ model.

of Piecuch, Oliphant and Adamowicz, 63 allowing a direct comparison. We investigate the system for $\alpha / a_{0} \in[0.0001,1]$ and the results are given in Figure 9. We find a noticeable

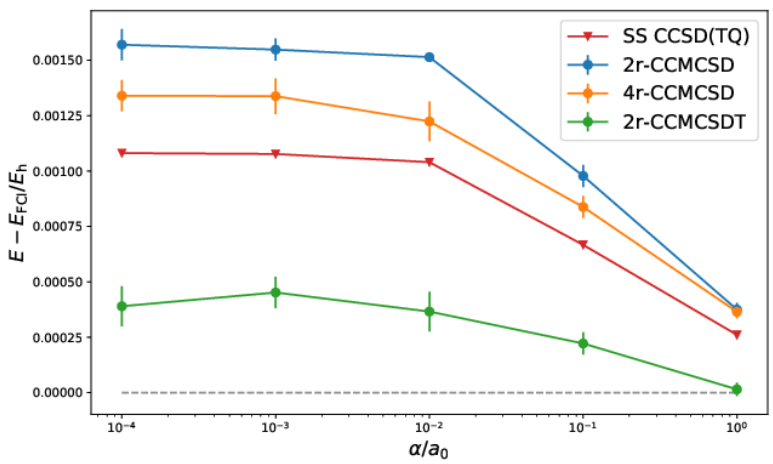

Figure 9: Comparison of single- and multireference coupled cluster results for the $\mathrm{H}_{8}$ model.

$(\approx 0.5$ miliHArtree $)$ discrepancy between $2 \mathrm{r}$ CCMC and SS CCSD(TQ) energies. Including the full $(2,2)$ CAS in the reference space decreases this discrepancy, but 4 -CCMCSD is still not in agreement with $\operatorname{SS~CCSD(TQ).~We~}$ expect this to be due to the absence of some terms in our cluster expansions compared to SS CCSD(TQ). To verify this, we compare the values given by these different approaches for the leading $\hat{T}_{3}$ terms in the CCSDTQ expansion.

As can be seen from Table 2, we observe more sign differences between the mr-CCSD wavefunctions and CCSDTQ than when comparing to SS CCSD(TQ). Also, at $\alpha=a_{0}$, the fifth largest triple excitation coefficient in CCSDTQ is on a term that is excluded from our calculations, but included in SS CCSD(TQ). The presence of such terms in the wavefunction at other geometries as well could explain the discrepancy between our methods and SS CCSD(TQ). Indeed if we look at the 2r-CCSDT wavefunction (which includes some pentuple contributions and therefore has significantly different cluster amplitudes than CCSDTQ), this cluster continues to make a significant contribution at $\alpha=0.1 a_{0}$. We can therefore expect such clusters to continue being significant in the CCSDTQ and SS CCSD(TQ) wavefunctions, potentially justifying the discrepancy with relative to 2r- and 4r-CCMCSD. While our method does not include them when considering the active space, a small number of additional references could be included to ensure their presence. Alternatively, increasing the truncation level to CCSDT in a two reference calculation is sufficient to recover these terms and indeed obtain much more accurate results than either mrCCMCSD or SS CCSD(TQ).

\subsubsection{The $\mathrm{N}_{2}$ molecule}

Figure 10 shows the difference between various implementations of MRCC, at CCSD level ${ }^{4 / 37}$ and the FCI energy along the $\mathrm{N}_{2}$ binding curve. It can be easily observed that 2r-CCMCSDT performs as well as the best of these methods, while 2r-CCMCSD shows a significant deviation from the FCI values. We believe that the primary cause of this is the fact that all conventional methods are built on top of a CASSCF calculation in the $\mathrm{N}_{2}(6,6)$ CAS,, 37 with all double excitations out of this CAS considered. It is immediately obvious that the spanned space of such calculations is a large superset of the space our two-reference CCSD calculation spans, which could be expected to improve the accuracy of these calculations.

To obtain a fairer comparison, we have included all 400 determinants in the CAS as references in our calculation (400r-CCSD), allowing double excitations out of each. Including the CAS in such a way is equivalent to using a CASCI reference wavefunction and significantly improves the quality of the obtained correlation energy (see Figure 10), yielding a method that outperforms all but the most accurate conventional methods. The remaining gap can be 
Table 2: Leading triply excited cluster coefficients in the $\mathrm{H}_{8}$ wavefunction for $\alpha / a_{0}=1.0,0.1,0.0001$.

a All wavefunctions are normalised such that $\left\langle D_{0} \mid \Psi\right\rangle=1$

b As given in 63

${ }^{\mathrm{c}}$ Values taken from instantaneous snapshots of the stochastic wavefunction.

\begin{tabular}{|c|c|c|c|c|c||c|}
\hline \multirow{2}{*}{$\alpha / a_{0}$} & \multirow{2}{*}{$\hat{T}_{3}$ term } & \multicolumn{5}{|c|}{ Coefficient $^{\mathrm{a}}$} \\
\cline { 3 - 7 } & & CCSDTQ $^{\mathrm{b}}$ & SS-CCSDTQ $^{\mathrm{b}}$ & 2r-CCMCSD $^{\mathrm{r}}$ & 4r-CCMCSD $^{\mathrm{c}}$ & 2r-CCMCSDT $^{\mathrm{c}}$ \\
\hline \multirow{5}{*}{1.0} & $t_{875}^{653}$ & 0.0027990300 & 0.0 & 0.0 & 0.0 & -0.006938820228 \\
& $t_{873}^{543}$ & -0.0027927466 & 0.0 & 0.0 & 0.0 & -0.005822500732 \\
& $t_{871}^{853}$ & 0.0026117601 & 0.0 & 0.0 & 0.0 & -0.00622210191 \\
& $t_{875}^{741}$ & -0.002605554 & -0.0026078696 & 0.004204674226 & 0.006681790253 & 0.005893986503 \\
& $t_{764}^{872}$ & 0.0025749909 & 0.0026218416 & 0.0 & 0.0 & -0.005829632611 \\
\hline \multirow{6}{*}{0.1} & $t_{875}^{321}$ & -0.0353278703 & -0.0304035827 & -0.02906899421 & -0.02533820583 & -0.03510182677 \\
& $t_{873}^{521}$ & 0.0341886609 & 0.0294198501 & -0.03320221354 & -0.2576256265 & -0.03417247948 \\
& $t_{763}^{721}$ & 0.0112172942 & 0.0091712186 & 0.009037433273 & 0.01719834927 & 0.01032064989 \\
& $t_{754}^{721}$ & -0.0109948657 & -0.0089897429 & -0.009720349583 & -0.009384567264 & 0.01176631082 \\
& $t_{732}^{521}$ & -0.0108251409 & -0.0087530913 & 0.007848827095 & 0.01093788654 & 0.005768813771 \\
& $t_{764}^{872}$ & - & - & 0.0 & 0.0 & 0.007187521769 \\
\hline
\end{tabular}

bridged by using CASSCF rather than HF orbitals, however this comes at an increased computational cost.

\subsubsection{The $\mathrm{H}_{2} \mathrm{O}$ molecule}

We also investigate the symmetric dissociation of the water molecule, over a range of $\mathrm{OH}$ bond lengths raging from the equilibrium value $R_{\mathrm{e}}=1.84345 a_{0}$ to $3 R_{\mathrm{e}}$, with the $\mathrm{HOH}$ angle fixed at $110.6 \mathrm{deg}$. As can be seen from Figure 11, for this system the CCSDT description fails at long bond lengths. By comparison, $\mathrm{CCSD}^{1}$ continues to provide reasonable descriptions across the binding curve. As in the case of $\mathrm{N}_{2}$, this molecule has been studied using state-specific MRCC methods, $\stackrel{37}{\text { based on }}$ the $(4,4) \mathrm{CASSCF}$ wavefunction as a reference. Both SS-MRCCSD and sr-MRBWCCSD consistently give errors of less than 5 and 15 miliHartree respectively, relative to the FCI results. The CCSDtq method has also been applied to this system, giving errors consistently below 3

\footnotetext{
${ }^{1}$ The performance of all is highly dependent of the exact Hartree-Fock reference used at $r_{\mathrm{OH}}=3 R_{\mathrm{e}}$, where there are two low lying RHF states. One, with $E=$ -75.34439Hartree, gives the results shown in Figure 11 for CCSD, CCSDT and mr-CCMC. The other, with $E=-75.4341998$ Hartree, causes both conventional CCSD and CCMCSD, CCMCSDT and mr-CCMCSDT to converge to a metastable excited state with $E_{\text {corr }} \approx$ -0.4 Hartree.
}

miliHartree, which can be reduced by applying further corrections. 16170

2r-CCMCSD, using the highest excited determinant in the $(4,4) \mathrm{CAS}$ as a secondary reference performs comparably to sr-MRBWCCSD, however 2r-CCMCSDT shows a significant improvement, with errors of less than 1.5 mHartrees across the entire binding curve. Unlike its single-reference counterpart, 2r-CCSDT provides a consistent description of the system at all bond lengths. As before, we can use all determinants in the $(4,4)$ CAS as references for a CCMCSD method. Once again, we observe a significant improvement in the quality of our estimates, generally outperforming conventional MRCC methods, but not 2r-CCMCSDT or CCSDtq.

\subsection{Bridging the gap}

While achieving results of similar quality for $\mathrm{N}_{2}$, it is worth noting that the stochastic Hilbert space of 2r-CCMCSDT is less than half of that of 400r-CCMCSD (68000 vs. 151100 determinants). A direct comparison of these Hilbert spaces shows that, rather than the 2r-CCMCSDT calculation spanning a strict subset of the 400r-CCMCSD space, they only partially overlap. The CAS shows significant redundancy in spanning this overlap, with an average of 9 CAS determinants connected to 

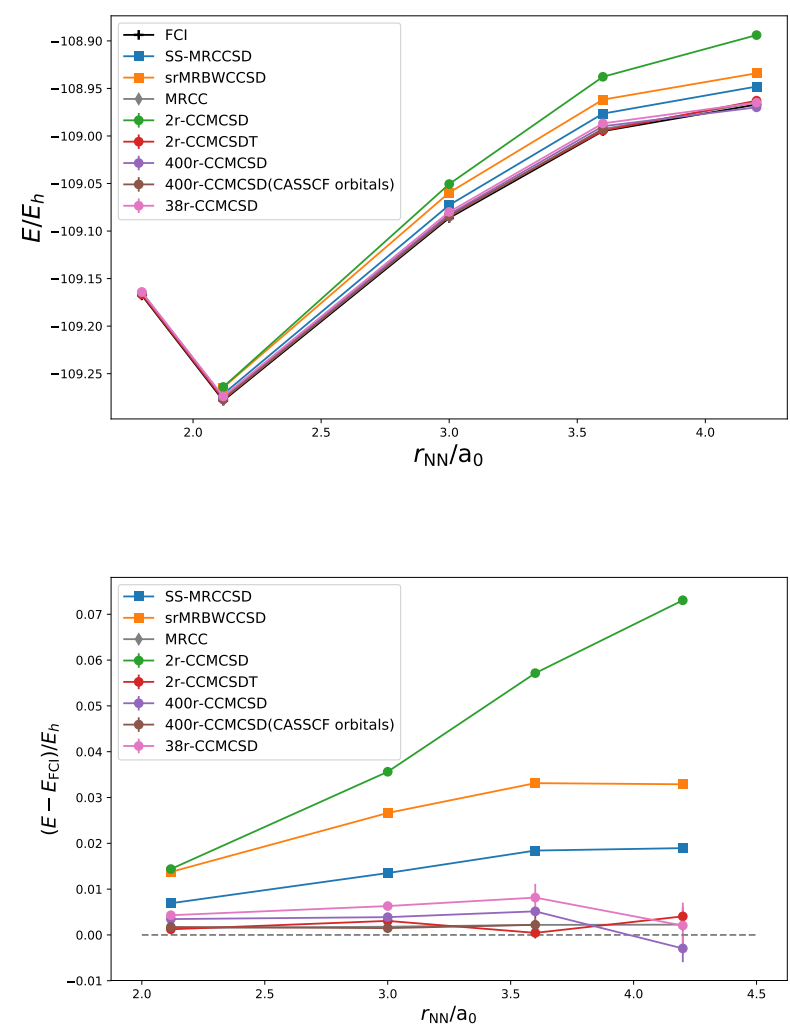

Figure 10: Comparison of $\mathrm{mrCC}$ and $\mathrm{mr-CCMC}$ methods to FCI along the $\mathrm{N}_{2}$ binding curve in the Dunning cc-pVDZ basis set. Conventional multireference results are from Ref. 4 and 37. While 2r-CCMCSD (based on either canonical HF or CASSCF orbitals) underperforms relative to these methods, accuracy is satisfactorily regained by increasing the number of references or moving to 2r-CCMCSDT.

any (connected) determinants. However, there are determinants in the overlap that are solely connected to one CAS determinant. Altogether these connect to only 38 of the CAS determinants and it turns out these 38 determinants are also sufficient to span the whole overlap. This suggests that the significant part of the wavefunction is encoded in this subspace. The flexibility mr-CCMC has in terms of defining references and their accepted cluster excitation levels allows us to easily investigate this hypothesis. Indeed, an mr-CCSD calculation using these 38 determinants as references recovers $98.7 \%$ of the correlation energy at $r=3.6 a_{0}$, while decreasing the Hilbert space (and therefore memory cost) by $82 \%$ compared to the 400r-CCSD case. It maintains this level of accuracy consistently across the binding curve, as

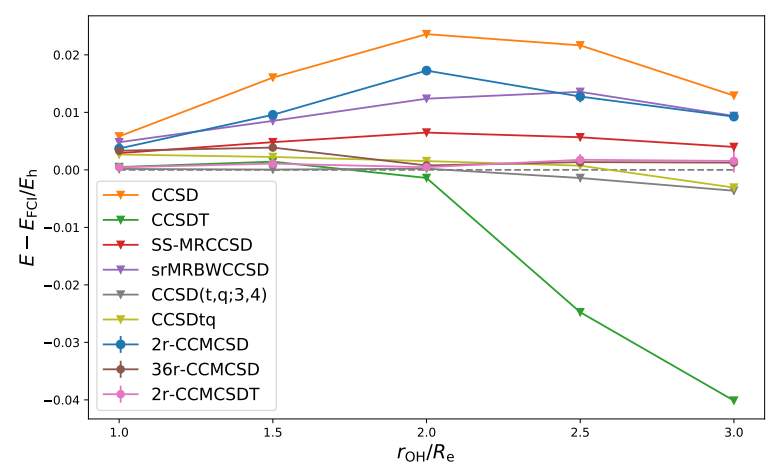

Figure 11: Coupled cluster energies of $\mathrm{H}_{2} \mathrm{O}$ relative to the FCI energy, during the symmetric stretch from $R_{\mathrm{OH}}=R_{\mathrm{e}}$ to $R_{\mathrm{OH}}=3 R_{\mathrm{e}}$, with the angle fixed at 110.6deg.

can be seen in Figure 10

The mr-CCMC method shows fast convergence of the correlation energy with increasing number of references from this subset (see Figure 12). While the exact details of the convergence depend on the order in which the references are included, the behaviour is significantly outside the standard deviation of a randomly selected set of 38 references, supporting the idea that these references and their excitations encode the significant part of the wavefunction. We also observe the expected sublinear scaling of memory cost with number of references, as their spawned spaces begin to overlap (see Figure 13).

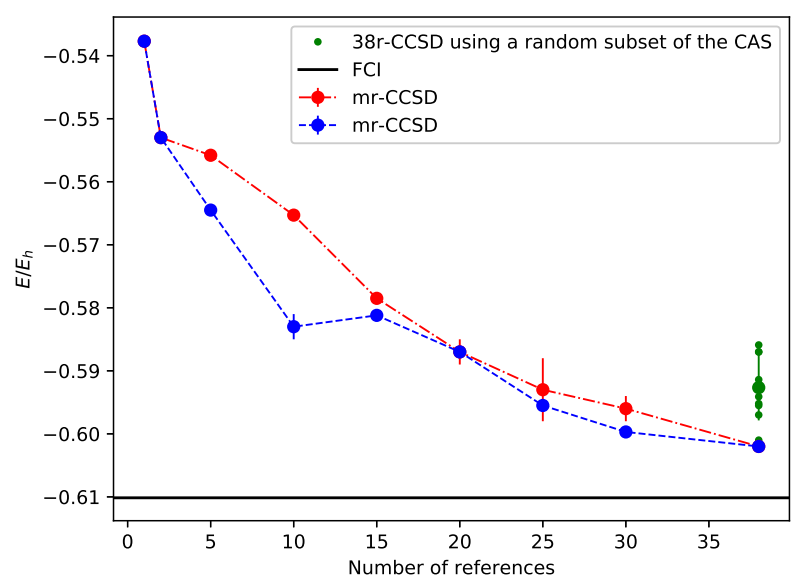

Figure 12: Convergence of the mr-CCMCSD energy with increasing number of references. The red and blue curves correspond to different orders of inclusion of references from the same 38-determinant set.

In order to obtain this optimised reference 


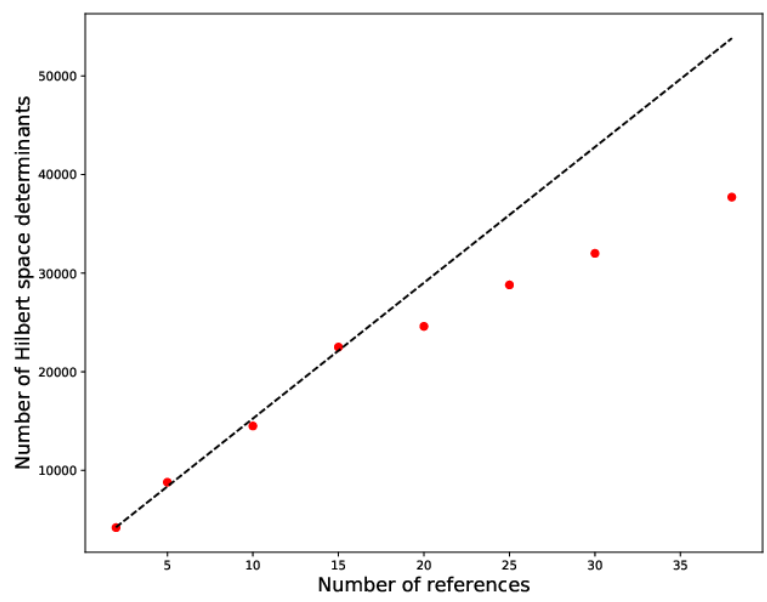

Figure 13: Variation of the size of the Hilbert space of a mr-CCMCSD calculation with the number of references for cc-pVDZ $\mathrm{N}_{2}$. As expected, the size of the Hilbert space increases sub-linearly with the number of references.

space, one requires knowledge of the spanned Hilbert spaces of the larger 400r-CCSD and 2rCCSDT calculations. In this work, the information was acquired from stochastic snapshots of the two calculations, however a list of all determinants in the Hilbert space of each calculation can be easily generated if the references and excitation levels of the methods are known. This could then be analysed in the same way we have done here and used to predict an optimised, less computationally expensive method, without incurring the cost of actually running the more demanding calculations.

\section{Conclusions}

We have successfully implemented a simple multireference technique within the framework of stochastic coupled cluster. The method shows a systematic improvement over singlereference CCMC, giving high-accuracy energy estimates in known strongly correlated molecular systems. The memory requirements are expected to scale sublinearly with the number of references used. This scaling is significantly better than the one expected with increasing the truncation level in a large Hilbert space, making the technique likely useful for the treatment of more complicated systems, with mul- tiple highly weighted determinants in the true ground state. Significantly, in most cases the performs at least as well as many deterministic multireference methods, while providing a simple algorithm and significant possibilities for expansion.

In one case, we have shown that lowered accuracy may be correlated to the absence of some potentially significant clusters from our expansion. The effect of including these clusters will be investigated further. We have also observed that the choice of one electron orbitals can affect the quality of the mr-CCMC results. We are interested in investigating iterative schemes to optimise the orbitals used.

The method also shows great flexibility in the choice of reference and excitation space used, without significant effects on the stability and general behaviour of the calculations. This allows for potential detailed investigation into the structure of coupled cluster wavefunctions, as well as potential optimised computations, using the minimal required reference space.

The extent to which the use of multiple references improves the correlation energy is system dependent, which may be at least partly due to the different quality of the secondary references. Therefore, a systematic way of selecting the best secondary references, especially in systems where chemical intuition is lacking, is of further interest. This could potentially be done by iteratively modifying the reference space, using an amplitude threshold, similarly to what is done in the initiator approach or selected CI. $71-75$ A connectivity criterion could also be implemented. With this refinement, we expect that this formulation of stochastic multireference coupled cluster could provide a flexible and robust method to compute accurate energies for a wide range of strongly correlated systems.

Acknowledgement M-A.F. is grateful to Magdalene College, Cambridge for summer project funding and to the Cambridge Trust and Corpus Christi College for a studentship. C.J.C.S. is grateful to the Sims Fund for a studentship and A.J.W.T. to the Royal Society for a University Research Fellowship under Grant No. UF160398. All are grateful for support un- 
der ARCHER Leadership Project grant e507. Molecular orbital integrals were generated using PySCF ${ }^{76}$ Psi4 ${ }^{77}$ and Q-Chem. ${ }^{78}$ CASSCF orbitals were obtained using PySCF or ORCA. ${ }^{79}$ Stochastic post-Hartree Fock and some FCI calculations were performed using a development version of HANDE-QMC .80 Deterministic CC calculations for $\mathrm{N}_{2}$ at $1.8 a_{0}$ were performed in MRCC. .81

\section{Supporting Information Avail- able}

Numerical values for CCMC energies, as well as Hartree-Fock and FCI references.

\section{References}

(1) Lyakh, D. I.; Musiał, M.; Lotrich, V. F.; Bartlett, R. J. Multireference Nature of Chemistry: The Coupled-Cluster View. Chem. Rev. 2011, 182.

(2) Čížek, J. On the Correlation Problem in Atomic and Molecular Systems. Calculation of Wavefunction Components in Ursell-Type Expansion Using QuantumField Theoretical Methods. J. Chem. Phys 1966, 45, 4256 .

(3) Č́žzek, J. On the Use of the Cluster Expansion and the Technique of Diagrams in Calculations of Correlation Effects in Atoms and Molecules. Adv. Chem. Phys. 1969, $24,35$.

(4) Chan, G. K.-L.; Kállay, M.; Gauss, J. State-of-the-art density matrix renormalization group and coupled cluster theory studies of the nitrogen binding curve. $J$. Chem. Phys. 2004, 121, 6110.

(5) Jeziorski, B.; Monkhorst, H. J. Coupledcluster method for multideterminantal reference states. Phys. Rev. A 1981, 24, 1668 .

(6) Piecuch, P.; Paldus, J. Orthogonally spinadapted multi-reference Hilbert space coupled-cluster formalism: diagrammatic formulation*. Theor. Chim. Acta 1992, 83, 69 .

(7) Piecuch, P.; Paldus, J. Orthogonally spinadapted state-universal coupled-cluster formalism: Implementation of the complete two-reference theory including cubic and quartic coupling terms. J. Chem. Phys 1994, 101, 5875 .

(8) Mahapatra, U. S.; Datta, B.; Mukherjee, D. A state-specific multi-reference coupled cluster formalism with molecular applications. Mol. Phys. 1998, 94, 157.

(9) Mahapatra, U. S.; Datta, B.; Mukherjee, D. A Size-Consistent State-Specific Multireference Coupled Cluster Theory: Formal Developments and Molecular Applications. J. Chem. Phys. 1999, 110, 6171 .

(10) Kowalski, K.; Piecuch, P. Extension of the method of moments of coupledcluster equations to a multireference wave operator formalism. J. Mol. Struct. THEOCHEM 2001, 547, 191.

(11) Chattopadhyay, S.; Pahari, D.; Mukherjee, D.; Mahapatra, U. S. A statespecific approach to multireference coupled electron-pair approximation like methods: Development and applications. J. Chem. Phys. 2004, 120, 5968.

(12) Hanrath, M. An exponential multireference wave-function Ansatz. J. Chem. Phys. 2005, 123, 084102.

(13) Mukherjee, D.; Kumar Moitra, R.; Mukhopadhyay, A. Correlation problem in open-shell atoms and molecules. Mol. Phys. 1975, 30, 1861.

(14) Oliphant, N.; Adamowicz, L. Multireference coupled-cluster method using a single-reference formalism. J. Chem. Phys. 1991, 94, 1229. 
(15) Piecuch, P.; Oliphant, N.; Adamowicz, L. A state-selective multireference coupledcluster theory employing the singlereference formalism. J. Chem. Phys 1993, 99, 1875 .

(16) Piecuch, P.; Kucharski, S. A.; Bartlett, R. J. Coupled-cluster methods with internal and semi-internal triply and quadruply excited clusters: CCSDt and CCSDtq approaches. J. Chem. Phys. 1999, 110, 1875.

(17) Kowalski, K.; Piecuch, P. The active-space equation-of-motion coupled-cluster methods for excited electronic states: The EOMCCSDt approach. J. Chem. Phys. 2000, 113, 1715.

(18) Lyakh, D. I.; Ivanov, V. V.; Adamowicz, L. Automated generation of coupledcluster diagrams: Implementation in the multireference state-specific coupledcluster approach with the complete-activespace reference. J. Chem. Phys. 2005, 122, 024108.

(19) Yanai, T.; Chan, G. K.-L. Canonical transformation theory for multireference problems. J. Chem. Phys. 2006, 124, 194106.

(20) Fang, T.; Li, S. Block correlated coupled cluster theory with a complete activespace self-consistent-field reference function: The formulation and test applications for single bond breaking. J. Chem. Phys. 2007, 127, 204108.

(21) Li, X.; Paldus, J. Partially linearized, fully size-extensive, and reduced multireference coupled-cluster methods. I. Formalism and mutual relationship. J. Chem. Phys. 2008, 128, 144118.

(22) Neuscamman, E.; Yanai, T.; Chan, G. K.L. Strongly contracted canonical transformation theory. J. Chem. Phys. 2010, 132, 024106 .
(23) Evangelista, F. A.; Gauss, J. An orbitalinvariant internally contracted multireference coupled cluster approach. J. Chem. Phys. 2011, 134, 114102.

(24) Shen, J.; Piecuch, P. Combining activespace coupled-cluster methods with moment energy corrections via the $\mathrm{CC}(\mathrm{P} ; \mathrm{Q})$ methodology, with benchmark calculations for biradical transition states. $J$. Chem. Phys 2012, 136, 144104.

(25) Mukherjee, D.; Kumar Moitra, R.; Mukhopadhyay, A. Applications of a non-perturbative many-body formalism to general open-shell atomic and molecular problems: calculation of the ground and the lowest $\pi-\pi^{*}$ singlet and triplet energies and the first ionization potential of transbutadiene. Mol. Phys. 1977, 33, 955.

(26) Haque, M. A.; Mukherjee, D. Application of cluster expansion techniques to openshells: Calculation of difference energies. J. Chem. Phys. 1984, 80, 5058.

(27) Lindgren, I.; Mukherjee, D. On the connectivity criteria in the open-shell coupled-cluster theory for general model spaces. Phys. Rep. 1987, 151, 93.

(28) Stolarczyk, L. Z.; Monkhorst, H. J. Coupled-cluster method in Fock space. I. General formalism. Phys. Rev. A 1985, 32, 725 .

(29) Kaldor, U. The Fock space coupled cluster method: theory and application. Theor. Chim. Acta 1991, 80, 427.

(30) Meissner, L. On multiple solutions of the Fock-space coupled-cluster method. Chem. Phys. Lett. 1996, 255, 244.

(31) Meissner, L. Fock-space coupled-cluster method in the intermediate Hamiltonian formulation: Model with singles and doubles. J. Chem. Phys. 1998, 108, 9227.

(32) Figgen, D.; Wedig, A.; Stoll, H.; Dolg, M.; Eliav, E.; Kaldor, U. On the performance 
of two-component energy-consistent pseudopotentials in atomic Fock-space coupled cluster calculations. J. Chem. Phys. 2008, $128,024106$.

(33) Li, X.; Paldus, J. The general-model-space state-universal coupled-cluster method exemplified by the LiH molecule. J. Chem. Phys. 2003, 119, 5346.

(34) Balková, A.; Kucharski, S. A.; Meissner, L.; Bartlett, R. J. A Hilbert space multi-reference coupled-cluster study of the $\mathrm{H}_{4}$ model system. Theor. Chim. Acta 1991, 80, 335 .

(35) Mukhopadhyay, D.; Mukherjee, D. Molecular applications of size-extensive quasi-Hilbert and quasi-Fock coupledcluster formalisms using incomplete model spaces. Chem. Phys. Lett. 1991, $177,441$.

(36) Balková, A.; Bartlett, R. J. A multireference coupled-cluster study of the ground state and lowest excited states of cyclobutadiene. J. Chem. Phys. 1994, 101, 8972.

(37) Das, S.; Mukherjee, D.; Kállay, M. Full implementation and benchmark studies of Mukherjee's state-specific multireference coupled-cluster ansatz. J. Chem. Phys. 2010, 132, 074103.

(38) Malrieu, J. P.; Durand, P.; Daudey, J. P. Intermediate Hamiltonians as a new class of effective Hamiltonians. J. Phys. A 1985, 18, 809 .

(39) Jankowski, K.; Malinowski, P. A valenceuniversal coupled-cluster single-and double-excitations method for atoms. III. Solvability problems in the presence of intruder states. J. Phys. B 1994, 27, 1287.

(40) Kaldor, U. Intruder states and incomplete model spaces in multireference coupledcluster theory: The $2 p^{2}$ states of Be. Phys. Rev. A 1988, 38, 6013.
(41) Paldus, J.; Piecuch, P.; Pylypow, L.; Jeziorski, B. Application of Hilbert-space coupled-cluster theory to simple $\left(\mathrm{H}_{2}\right)_{2}$ model systems: Planar models. Phys. Rev. A 1993, 47, 2738.

(42) Piecuch, P.; Toboła, R.; Paldus, J. Approximate account of connected quadruply excited clusters in multi-reference Hilbert space coupled-cluster theory. Application to planar $\mathrm{H}_{4}$ models. Chem. Phys. Lett. 1993, 210, 243.

(43) Piecuch, P.; Paldus, J. Application of Hilbert-space coupled-cluster theory to simple $\left(\mathrm{H}_{2}\right)_{2}$ model systems. II. Nonplanar models. Phys. Rev. A 1994, 49, 3479.

(44) Kállay, M.; Szalay, P. G.; Surján, P. R. A general state-selective multireference coupled-cluster algorithm. J. Chem. Phys. 2002, 117, 980 .

(45) Nooijen, M.; Shamasundar, K. R.; Mukherjee, D. Reflections on sizeextensivity, size-consistency and generalized extensivity in many-body theory. Mol. Phys. 2005, 103, 2277.

(46) Lyakh, D. I.; Ivanov, V. V.; Adamowicz, L. A generalization of the statespecific complete-active-space coupledcluster method for calculating electronic excited states. J. Chem. Phys. 2008, 128, 074101.

(47) Booth, G. H.; Thom, A. J. W.; Alavi, A. Fermion Monte Carlo without fixed nodes: A game of life, death, and annihilation in Slater determinant space. J. Chem. Phys. 2009, 131, 054106.

(48) Cleland, D.; Booth, G. H.; Alavi, A. Communications: Survival of the fittest: Accelerating convergence in full configuration-interaction quantum Monte Carlo. J. Chem. Phys. 2010, 132, 041103.

(49) Booth, G. H.; Cleland, D.; Thom, A. J. W.; Alavi, A. Breaking the carbon dimer: The challenges of multiple bond 
dissociation with full configuration interaction quantum Monte Carlo methods. J. Chem. Phys. 2011, 135, 084104.

(50) Shepherd, J. J.; Booth, G.; Grüneis, A.; Alavi, A. Full configuration interaction perspective on the homogeneous electron gas. Phys. Rev. B 2012, 85, 081103.

(51) Booth, G. H.; Grüneis, A.; Kresse, G.; Alavi, A. Towards an exact description of electronic wavefunctions in real solids. $\mathrm{Na}$ ture 2013, 493, 365.

(52) Thom, A. J. W. Stochastic Coupled Cluster Theory. Phys. Rev. Lett. 2010, 105, 263004.

(53) Deustua, J. E.; Shen, J.; Piecuch, P. Converging High-Level Coupled-Cluster Energetics by Monte Carlo Sampling and Moment Expansions. Phys. Rev. Lett 2017, 119, 223003.

(54) Deustua, J. E.; Magoulas, I.; Shen, J.; Piecuch, P. Communication: Approaching exact quantum chemistry by cluster analysis of full configuration interaction quantum Monte Carlo wave functions. $J$. Chem. Phys. 2018, 149, 151101.

(55) Spencer, J. S.; Thom, A. J. W. Developments in stochastic coupled cluster theory: The initiator approximation and application to the uniform electron gas. J. Chem. Phys. 2016, 144, 084108.

(56) Spencer, J. S.; Neufeld, V. A.; Vigor, W. A.; Franklin, R. S. T.; Thom, A. J. W. Large scale parallelization in stochastic coupled cluster. $J$. Chem. Phys. 2018, 149, 204103.

(57) Scott, C. J. C.; Thom, A. J. W. Stochastic coupled cluster theory: Efficient sampling of the coupled cluster expansion. J. Chem. Phys. 2017, 147, 124105.

(58) Franklin, R. S. T.; Spencer, J. S.; Zoccante, A.; Thom, A. J. W. Linked coupled cluster Monte Carlo. J. Chem. Phys. 2016, 144, 044111.
(59) Holmes, A. A.; Changlani, H. J.; Umrigar, C. J. Efficient Heat-Bath Sampling in Fock Space. J. Chem. Theory Comput. 2016, 12, 1561.

(60) Neufeld, V. A.; Thom, A. J. W. Exciting Determinants in Quantum Monte Carlo: Loading the Dice with Fast, Low Memory Weights. J. Chem. Theory Comput. 2019, $15,127$.

(61) Scott, C. J. C.; Di Remigio, R.; Crawford, T. D.; Thom, A. J. W. Diagrammatic Coupled Cluster Monte Carlo. J. Phys. Chem. Lett 2019, 10, 925.

(62) Oliphant, N.; Adamowicz, L. The implementation of the multireference coupledcluster method based on the singlereference formalism. J. Chem. Phys. 1992, 96, 3739 .

(63) Piecuch, P.; Adamowicz, L. State-selective multireference coupled-cluster theory employing the single-reference formalism: Implementation and application to the $\mathrm{H}_{8}$ model system. J. Chem. Phys 1994, 100, 5792 .

(64) Jankowski, K.; Paldus, J. Applicability of coupled-pair theories to quasidegenerate electronic states: A model study. Int. J. Quantum Chem. 1980, 18, 1243.

(65) Laidig, W. D.; Saxe, P.; Bartlett, R. J. The Description of $\mathrm{N}_{2}$ and $\mathrm{F}_{2}$ potential energy surfaces using multireference coupled cluster theory. J. Chem. Phys. 1987, $86,887$.

(66) Hehre, W. J.; Stewart, R. F.; Pople, J. A. Self-Consistent Molecular-Orbital Methods. I. Use of Gaussian Expansions of Slater-Type Atomic Orbitals. J. Chem. Phys. 1969, 51, 2657.

(67) Dunning, T. H. Gaussian basis sets for use in correlated molecular calculations. I. The atoms boron through neon and hydrogen. J. Chem. Phys. 1989, 90, 1007. 
(68) Jones, K. The Chemistry of Nitrogen: Pergamon Texts in Inorganic Chemistry; Elsevier, 2016.

(69) Jankowski, K.; Meissner, L.; Wasilewski, J. Davidson-type corrections for quasidegenerate states. Int. J. Quantum Chem. 1985, 28, 931-942.

(70) Bauman, N. P.; Shen, J.; Piecuch, P. Combining active-space coupled-cluster approaches with moment energy corrections via the $\mathrm{CC}(\mathrm{P} ; \mathrm{Q})$ methodology: connected quadruple excitations. Mol. Phys. 2017, $115,2860$.

(71) Huron, B.; Malrieu, J. P.; Rancurel, P. Iterative perturbation calculations of ground and excited state energies from multiconfigurational zeroth-order wavefunctions. J. Chem. Phys. 1973, 58, 5745.

(72) Evangelisti, S.; Daudey, J.-P.; Malrieu, J.P. Convergence of an improved CIPSI algorithm. Chem. Phys. 1983, 75, 91.

(73) Tubman, N. M.; Lee, J.; Takeshita, T. Y.; Head-Gordon, M.; Whaley, K. B. A deterministic alternative to the full configuration interaction quantum Monte Carlo method. J. Chem. Phys. 2016, 145, 044112 .

(74) Holmes, A. A.; Tubman, N. M.; Umrigar, C. J. Heat-Bath Configuration Interaction: An Efficient Selected Configuration Interaction Algorithm Inspired by Heat-Bath Sampling. J. Chem. Theory Comput. 2016, 12, 3674.

(75) Schriber, J. B.; Evangelista, F. A. Communication: An adaptive configuration interaction approach for strongly correlated electrons with tunable accuracy. J. Chem. Phys. 2016, 144, 161106.

(76) Sun, Q.; Berkelbach, T. C.; Blunt, N. S.; Booth, G. H.; Guo, S.; Li, Z.; Liu, J.; McClain, J. D.; Sayfutyarova, E. R.;
Sharma, S.; et al, PySCF: the Pythonbased Simulations of Chemistry Framework. WIREs Comput. Mol. Sci. 2017, 8, e1340.

(77) Parrish, R. M.; Burns, L. A.; Smith, D. G. A.; Simonett, A. C.; DePrince III, A. E.; Hohenstein, E. G.; Bozkaya, U.; Sokolov, A. Y.; Di Remigio, R.; Richard, R. M.; Gonthier, J. F.; et al, Psi4 1.1: An Open-Source Electronic Structure Program Emphasizing Automation, Advanced Libraries, and Interoperability. J. Chem. Theory Comput 2017, 13, 3185 .

(78) Shao, Y.; Gan, Z.; Epifanovsky, E.; Gilbert, A. T. B.; Wormit, M.; Kussmann, J.; Lange, A. W.; Behn, A.; Deng, J.; Feng, X. 1. Advances in Molecular Quantum Chemistry Contained in the Q-Chem 4 Program Package. Mol. Phys. 2015, 113, 184.

(79) Neese, F. The ORCA program system. WIREs Comput. Mol. Sci. 2012, 2, 7378.

(80) Spencer, J. S.; Blunt, N. S.; Choi, S.; Etrych, J.; Filip, M.-A.; Foulkes, W. M. C.; Franklin, R. S. T.; Handley, W. J.; Malone, F. D.; Neufeld, V. A.; et al, The HANDE-QMC Project: Open-Source Stochastic Quantum Chemistry from the Ground State Up. J. Chem. Theory Comput. 2019, 15, 1728.

(81) Kállay, M.; Rolik, Z.; Csontos, J.; Nagy, P.; Samu, G.; Mester, D.; Csóka, J.; Szabó, B.; Ladjánszki, I.; Szegedy, L.; et al, MRCC, a Quantum Chemical Program Suite. www.mrcc.hu. 